\title{
High-Performance Liquid Chromatographic Determination and Metabolic Study of Sennoside A in Daiokanzoto by Mouse Intestinal Bacteria
}

\author{
Kento Takayama, Emi Matsui, Takeshi Kobayashi, Hirofumi Inoue, Yasuto Tsuruta, and \\ Nobuyuki OKAMURA* \\ Faculty of Pharmacy and Pharmaceutical Sciences, Fukuyama University; 1 Sanzo, Gakuen-cho, Fukuyama, Hiroshima \\ 729-0292, Japan. Received March 14, 2011; accepted June 5, 2011; published online June 6, 2011
}

\begin{abstract}
Daiokanzoto (DKT, combination of rhubarb and glycyrrhiza), a Kampo medicine, is clinically effective for constipation. Sennoside A is well known to induce diarrhea. Sennoside A is a prodrug that is transformed into an active metabolite, rheinanthrone, by intestinal bacteria. In this study, we investigated the effects of glycyrrhiza on the activity of sennoside A metabolism in intestinal bacteria using mouse feces. A high-performance liquid chromatography (HPLC) method for the determination of sennoside $A$ in incubation mixture of DKT with mouse feces was established. The retention time of sennoside A was $\mathbf{9 . 2 6 \pm 0 . 0 2}$ min with a TSKgel ODS-80TsQA column by linear gradient elution using a mobile phase containing aqueous phosphoric acid and acetonitrile and detection at $265 \mathrm{~nm}$. We found that the activity of sennoside A metabolism in intestinal bacteria was significantly accelerated when glycyrrhiza, liquiritin or liquiritin apioside coexisted with sennoside A, whereas that of glycyrrhizin was not altered. This method is applicable for determination of the activity of sennoside A metabolism by anaerobic incubation of DKT with mouse feces.
\end{abstract}

Key words daiokanzoto; sennoside A; metabolism; glycyrrhiza; liquiritin; liquiritin apioside

Kampo is a Japanese traditional medicine that was developed from traditional medicine originating in ancient China. Kampo medicines are generally produced by the combination of various crude drugs containing many constituents that have additive and/or synergistic effects or minimize harmful reactions. This characteristic makes it extremely difficult to reveal the mechanism of the pharmacological action. Kampo medicines are orally administered, and they contain many kinds of glycosides as the main and/or active constituents. The glycosides are not absorbed and brought into the lower part of the intestine. Most glycosides may be metabolized by intestinal bacteria producing $\beta$-D-glucosidases before being absorbed into the body. Therefore, the intestinal bacteria related to the metabolism of glycosides administered orally are important to understand the pharmacological effects. ${ }^{1-3)}$

Daiokanzoto (DKT) is the most well-known Kampo medicine and has been used for the treatment of constipation. DKT consists of Rhei Rhizoma (rhubarb) and Glycyrrhizae Radix (glycyrrhiza). Rhubarb and sennoside A, one of the main constituents of rhubarb, are well known to induce diarrhea. Glycyrrhiza is a crude drug contained in more than $70 \%$ of Kampo medicines certified by the Japanese Ministry of Health and Welfare. ${ }^{4}$ Glycyrrhiza has been considered to moderate the effects of other crude drugs in Kampo medicine. Glycyrrhiza showed a significant potentiating effect on the purgative action of rhubarb in rats when DKT was prepared as a proportion of rhubarb 4 to glycyrrhiza $1 .^{5)}$ This potentiating effect is mainly due to the enhanced secretion of water in the colon. In DKT, glycyrrhiza and liquiritin, the main constituent of glycyrrhiza, cooperatively prevent the rhubarb-induced strong spiking activity of colonic circular mucus. ${ }^{6)}$

Sennoside A is an inactive glycoside and it is a prodrug that is transformed into an active metabolite, rheinanthrone, by intestinal bacteria. ${ }^{7-9)}$ Studies on the metabolism of sennoside A in DKT contribute to the understanding of the phar- macological action of the drugs now in use. Although we developed an HPLC method for simultaneous determination of sennoside A metabolites in studies on the metabolism of sennoside A with rat and mouse feces, ${ }^{10)}$ rheinanthrone was unstable in the incubation mixtures of rhubarb. Therefore, sennoside A was used as a marker compound in this study on the metabolism of sennoside A. HPLC methods for the determination of sennoside $\mathrm{A}$ in rhubarb have been reported, ${ }^{11-14}$ but these methods were not applicable to the determination of sennoside $\mathrm{A}$ in the incubation mixtures of DKT because of interferences from the other metabolites present.

In this report, a reliable and simple HPLC method for determination of sennoside A after anaerobic incubation of DKT with mouse fecal flora is described. In addition, to elucidate the role of glycyrrhiza in DKT, we investigated the effect of constituents in glycyrrhiza on the purgative action of sennoside A using this method.

\section{Experimental}

Materials The chopped crude drugs in DKT, rhubarb (kinmon-daio in Japanese) and glycyrrhiza (touhoku-kanzo in Japanese), were purchased from Tochinototenkaido (Osaka, Japan). Sennoside A and glycyrrhizin were purchased from Wako Pure Chemical Industries (Osaka, Japan). Liquiritin and liquiritin apioside were used as authentic samples. Ultrapure distilled water was prepared with deionized-distilled water. All other chemicals were analytical reagent- or HPLC-grade commercial products.

Animal Preparation Animal experiments were carried out in accordance with the Guidelines for Animal Experimentation of Fukuyama University. Male ddy mice, weighing $30-40 \mathrm{~g}$, were obtained from SHIMIZU Laboratory Supplies (Kyoto, Japan) and housed in a $12 \mathrm{~h}$ light-dark cycle at 21 to $24^{\circ} \mathrm{C}$ for at least one week before the experiments. They were given free access to food and water throughout the study.

Chromatographic Condition The HPLC apparatus was an Agilent Technologies 1100 Series system (Waldbronn, Germany) consisting of a binary pump, an autosampler, a thermostated column compartment and a photodiode array (PDA) detector. All modules and data collection were controlled by Agilent Chemstation software. The column was a TSKgel ODS80TsQA reversed-phase column, particle size of the packing $5 \mu \mathrm{m}$, $150 \times 4.6 \mathrm{~mm}$ i.d. (Tosoh, Tokyo, Japan). The mobile phase was a gradient system with $200 \mu \mathrm{l} \cdot 1^{-1}$ (ca. $\left.0.017 \%\right)$ phosphoric acid in water (A) and ace- 
tonitrile (B), which were deaerated by sonication before use. Elution program was performed from 19 to $20 \%$ B for $3 \mathrm{~min}, 20$ to $21 \%$ B for $5 \mathrm{~min}$, a stepwise increase to $38 \% \mathrm{~B}$ for $0 \mathrm{~min}, 38$ to $42 \% \mathrm{~B}$ for $8 \mathrm{~min}$ and a stepwise increase to $90 \% \mathrm{~B}$ for $4 \mathrm{~min}$ to wash. A re-equilibration period of $12 \mathrm{~min}$ was used between individual runs. The flow rate was $1.0 \mathrm{ml} \cdot \mathrm{min}^{-1}$. The injection volume was $5 \mu \mathrm{l}$. The column temperature was $50^{\circ} \mathrm{C}$. The detection wavelength was set at $265 \mathrm{~nm}$ for determination and in the range of 200 to $500 \mathrm{~nm}$ for validation of peak purity.

Assay of Activity of Sennoside A Metabolism by Mouse Intestinal Bacteria Fresh feces obtained from mice were homogenized in 5 volumes of $0.01 \mathrm{M}$ potassium phosphate buffer ( $\mathrm{pH} 7.4$ ) by bubbling with $\mathrm{CO}_{2}$ gas to eliminate air. The fecal suspension was centrifuged at $1500 \boldsymbol{g}$ for $1 \mathrm{~min}$ and the supernatant was used as an intestinal bacterial mixture. Tubes containing sennoside A $(0.375 \mathrm{mg})$ in $0.01 \mathrm{M}$ potassium phosphate buffer $(0.25 \mathrm{ml})$ and a fecal suspension $(1 \mathrm{ml})$ were incubated at $37^{\circ} \mathrm{C}$ for different incubation periods $(2,4,8 \mathrm{~h})$ under anaerobic conditions. Anaerobic procedures were carried out using an anaerobic jar with an AnaeroPack (Mitsubishi Gas Chemical, Tokyo, Japan). The reaction was immediately stopped by adding $0.425 \%$ $\mathrm{v} / \mathrm{v}$ phosphoric acid in methanol $(1.25 \mathrm{ml})$. After centrifugation at $1500 \boldsymbol{g}$ for 5 min, the supernatant was passed through Minisart RC 15 (Japan Sartorius, Tokyo, Japan) and subjected to HPLC. The control process involved an incubation mixture at $0 \mathrm{~min}$, as mentioned above. Metabolic rate was calculated by the percentage of the content of sennoside $\mathrm{A}$ in the incubation mixture compared with that in the control.

Preparation of Calibration Curve Sennoside A was accurately weighed and dissolved in $0.2 \mathrm{M}$ ammonia-methanol to prepare a concentration of $6 \mathrm{mg} / \mathrm{ml}$ (stock solution). Test solutions were prepared followed by continuous dilution of the stock solution with methanol to give the twelve respective concentrations $(1.7 \mu \mathrm{M}$ to $3.48 \mathrm{~mm})$. Linearities were examined using two different standard solutions. Standard solution I was prepared from the test solutions $(0.5 \mathrm{ml})$ by mixing with $0.01 \mathrm{M}$ potassium phosphate buffer $(1.25 \mathrm{ml})$ and $0.708 \% \mathrm{v} / \mathrm{v}$ phosphoric acid in methanol $(0.75 \mathrm{ml})$. Standard solution II was prepared from the test solutions $(0.5 \mathrm{ml})$ by mixing with water $(1.25 \mathrm{ml})$-methanol $(0.75 \mathrm{ml})$. Standard solution was filtered through a Minisart-RC 15 filter and subjected to HPLC. The calibration curves were analyzed using linear regression derived from the peak area of sennoside A with three injections for each concentration.

Recovery Tests Incubation mixtures were prepared by incubating sennoside A $(0.05 \mathrm{mg})$, DKT $(5 \mathrm{mg})$ or sennoside A-glycyrrhiza $(0.375$, $4.25 \mathrm{mg}$, respectively) in $0.01 \mathrm{M}$ potassium phosphate buffer $(0.25 \mathrm{ml})$ and a fecal suspension $(1 \mathrm{ml})$ at $37^{\circ} \mathrm{C}$ for $4 \mathrm{~h}$ under anaerobic conditions. The incubation mixtures spiked with various concentrations of test solutions $(0.5 \mathrm{ml})$ were immediately mixed with $0.708 \% \mathrm{v} / \mathrm{v}$ phosphoric acid in methanol $(0.75 \mathrm{ml})$. After centrifugation, the supernatant was passed through a Minisart RC 15 and subjected to HPLC. Spiked samples were then analyzed in triplicate. The recoveries of sennoside A were determined from the slope ratios of regression equations with/without the incubation mixture using standard solution II.

Precision The within-day and day-to-day precision of the method for determination of sennoside A was evaluated using standard solution II and DKT. The test solution $(0.5 \mathrm{ml})$ diluted 16 times was mixed with $0.01 \mathrm{M}$ potassium phosphate buffer $(1.25 \mathrm{ml})$ and $0.708 \% \mathrm{v} / \mathrm{v}$ phosphoric acid in methanol $(0.75 \mathrm{ml})$. DKT $(5 \mathrm{mg})$ in $0.01 \mathrm{M}$ potassium phosphate buffer $(0.25 \mathrm{ml})$ was added to a mixture of fecal suspension $(1 \mathrm{ml})$ and $0.425 \% \mathrm{v} / \mathrm{v}$ phosphoric acid in methanol $(1.25 \mathrm{ml})$. The within-day precision was examined with fifteen replicate assays on the same day. The day-to-day precision was assessed by analyzing a mixture stored at $4{ }^{\circ} \mathrm{C}$ on five different days over two weeks. All sample solutions were filtered through a Minisart-RC 15 filter and subjected to HPLC.

Statistical Analyses Data are shown as the mean \pm S.D. The statistical significance of differences among values was examined by Dunnett's test. A probability value of $p<0.05$ was considered to indicate statistical significance.

\section{Results and Discussion}

Separation of Sennoside A in Incubation Mixture of DKT We have been studying metabolic study of glycyrrhizin using rat and mouse feces. ${ }^{15-17)}$ To elucidate the role of glycyrrhiza in DKT, the influence of glycyrrhiza and glycyrrhiza constituents on the activity of sennoside A metabolism was investigated using mouse feces. HPLC methods for the determination of sennoside $\mathrm{A}$ in rhubarb have been developed. ${ }^{11-14)}$ However, these methods were not applicable to the determination of sennoside $\mathrm{A}$ in incubation mixtures of DKT, because metabolites derived from glycyrrhiza constituents interfered with the measurement of sennoside A. We have reported an HPLC method for determination of sennoside $\mathrm{A}$ in Kampo medicines containing rhubarb ${ }^{18)}$; however, it is difficult to measure sennoside $\mathrm{A}$ in incubation mixtures of DKT because sennoside A cannot be separated from other metabolites. Several columns were preliminarily tested. The adequate separation of sennoside $\mathrm{A}$ was achieved with TSKgel ODS-80TsQA reversed-phase column as shown in Fig. 1. Analysis of the incubation mixture was performed using an HPLC apparatus equipped with a PDA detector. UV data of the effluent from the column ranging from 200 to $500 \mathrm{~nm}$ were collected. Sennoside A has a maximum UV absorbance at $265 \mathrm{~nm}$. The optimum monitoring wavelength for quantification was set at $265 \mathrm{~nm}$. When DKT with a fecal suspension was incubated at $37^{\circ} \mathrm{C}$ for $4 \mathrm{~h}$ under anaerobic conditions, the peak of sennoside A almost disappeared. Sennoside A was clearly separated without any pre-purification and determined within $20 \mathrm{~min}$ without interference from coexisting components. Phosphoric acid (ca. 0.017\% v/v) in the mobile phase affected the increase of the capacity factor of sennoside A, which has a carboxylic moiety. The retention time (capacity factor, $\left.k^{\prime}\right)$ was $9.24 \pm 0.02 \min \left(k^{\prime}: 4.84\right)$. The relative standard deviation (RSD, $n=10)$ of the retention time was $0.24 \%$. The peak was identified by comparison with an authentic specimen on inspections of the retention time and the UV spectrum, and purity was checked by three-dimensional chromatography.

Calibration Curve, Recovery Test and Precision When standard solution I and standard solution II were prepared, sennoside A precipitated in standard solution I in a high-concentration range. The slopes of regression equations of standard solution I and standard solution II were examined. However, the slope of the regression equation of standard solution II obtained at a wide range of concentration was almost the same as that of standard solution I obtained at the low-concentration range. In this study, the standard curve obtained from standard solution II was used. Linearity was evaluated over two ranges of 3.40 to $440 \mu \mathrm{M}$ and 108.7 to $1740 \mu \mathrm{M}$. The regression equations (correlation coefficients)

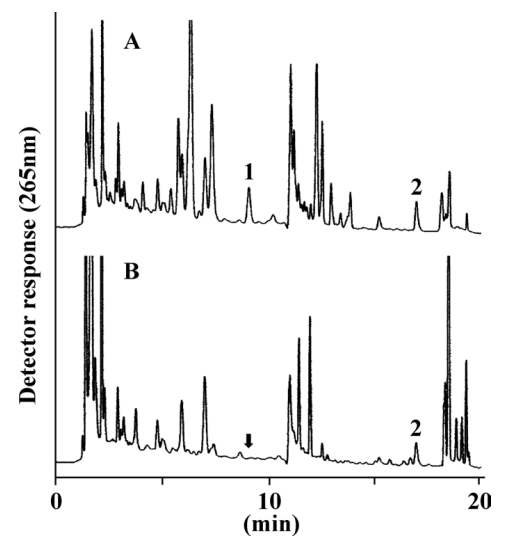

Fig. 1. HPLC Profiles of (A) Daiokanzoto and (B) Daiokanzoto Incubation Mixture

Peaks: 1, sennoside A; 2, glycyrrhizin. 
were $y=0.36 x-0.13(1.0000)$ and $y=0.36 x+0.39(0.9998)$, respectively, where $y$ is the peak area and $x$ is the concentration $(\mu \mathrm{M})$. The recovery was examined using incubation mixtures of sennoside A (Fig. 2A), DKT (Fig. 2B) or glycyrrhiza (Fig. 2C) spiked with various concentrations of test solutions (52.3 to $418.6 \mu \mathrm{M}, 102.4$ to $871.7 \mu \mathrm{M}$ ) after incubation at $37^{\circ} \mathrm{C}$ for $4 \mathrm{~h}$. The relationships between the peak areas and the concentrations were linear. The recoveries obtained were $100.8,97.5$ and $94.4 \%$, respectively. The detection limit of sennoside A (signal-to-noise ratio=3) was 8.5 pmol per injection $(5 \mu \mathrm{l})$.

The within-day and day-to-day precisions of the method for determination were evaluated using standard solution II and the incubation mixture of DKT. The within-day precision was examined with fifteen replicate assays per day and the day-to-day precision by assays on five different days. As shown in Table 1, the within-day and day-to-day relative standard deviations were 0.25 to $1.31 \%$.

Effect of Glycyrrhiza on Activity of Sennoside A Metabolism Glycyrrhiza is one of the most frequently used crude drugs in Kampo medicines. To investigate the effects of glycyrrhiza in DKT, the investigation of sennoside A metabolism, to which glycyrrhiza was added, was carried out. In Fig. 3, the mean time courses of metabolic ratio of sennoside $\mathrm{A}$ are shown. At more than $2 \mathrm{~h}$ of incubation, sennoside $\mathrm{A}$ was significantly metabolized in the presence of glycyrrhiza compared with the case in the absence of glycyrrhiza (Fig. 3A). Although glycyrrhizin is known as one of the active saponins of glycyrrhiza, it showed no influence on the metabolic ratio of sennoside A (Fig. 3B). On the other hand, the
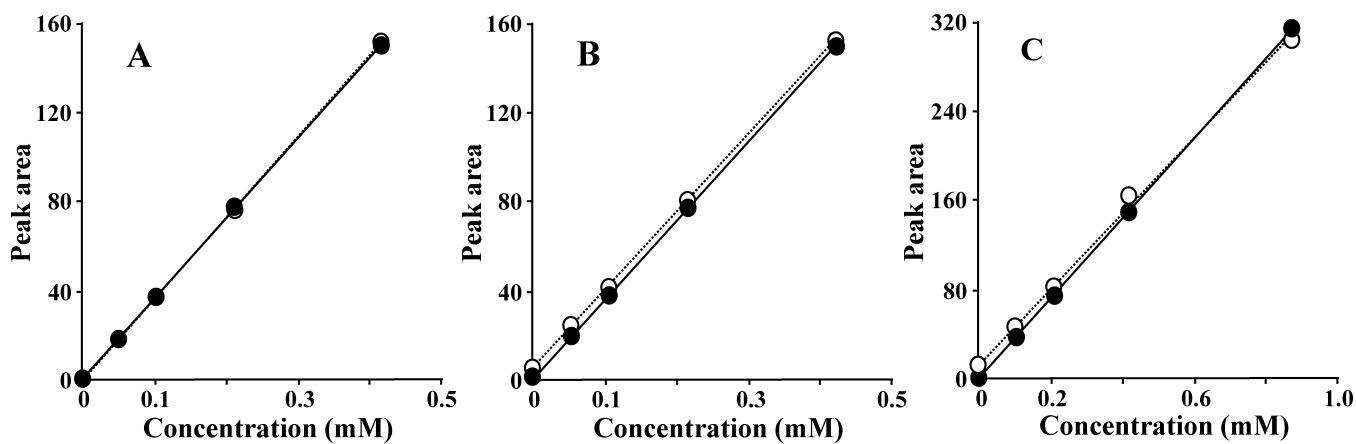

Fig. 2. Recovery Test of Sennoside A with and without Incubation Mixture of (A) Sennoside A, (B) Daiokanzoto and (C) Glycyrrhiza

Open circles: sennoside A with incubation mixtures. Closed circles: sennoside A without incubation mixtures. (A) Open circles: $y=0.36 x-0.96, r=0.9998$, closed circles: $y=0.36 x-0.13, r=1.0000$. (B) Open circles: $y=0.35 x+4.98, r=0.9999$, closed circles: $y=0.36 x-0.13, r=1.0000$. (C) Open circles: $y=0.34 x+10.24, r=0.9984$, closed circles: $y=0.36 x+0.39, r=0.9998$
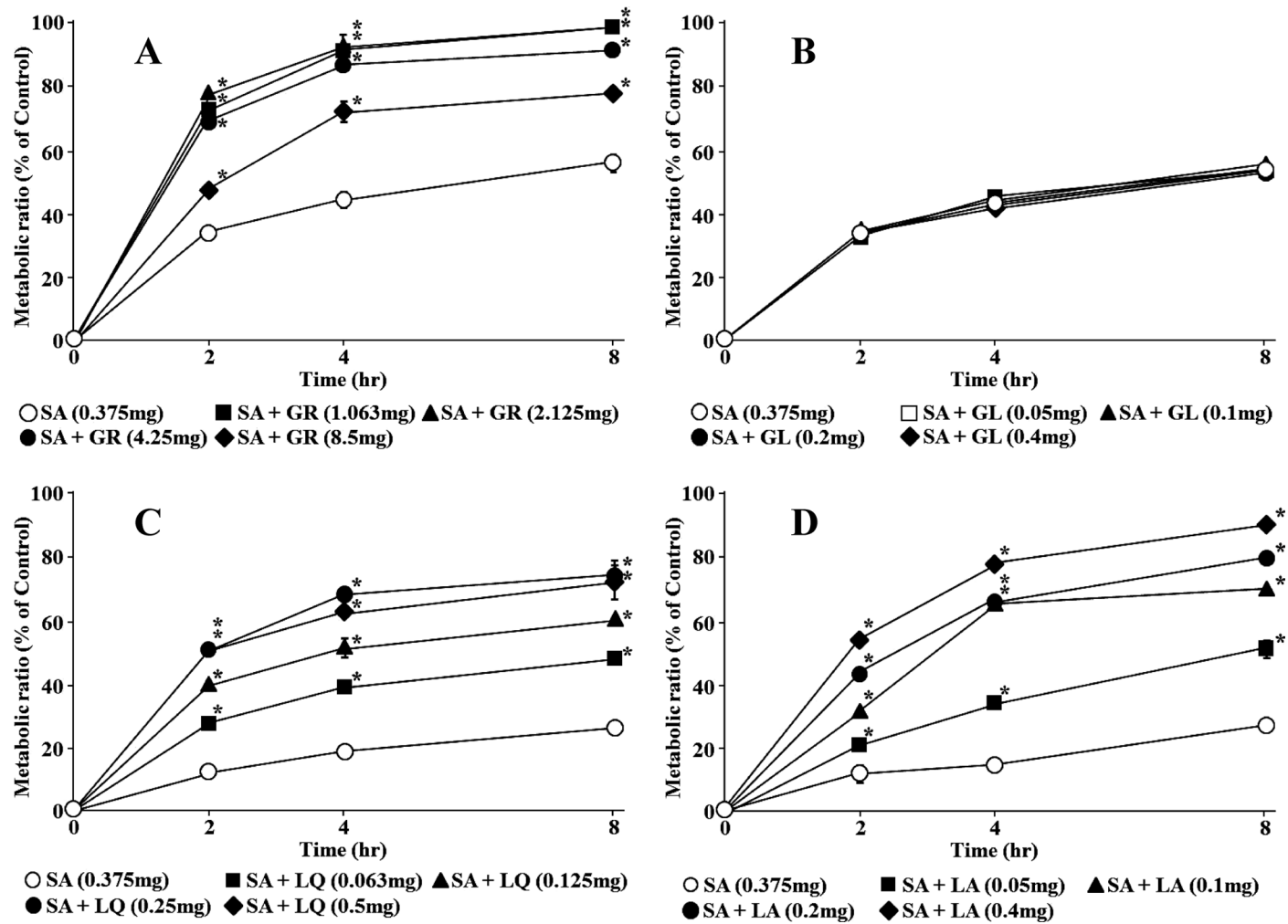

Fig. 3. Accelerating Effects of (A) Glycyrrhiza (GR), (B) Glycyrrhizin (GL), (C) Liquiritin (LQ) and (D) Liquiritin Apioside (LA) on Metabolism of Sennoside A (SA)

Each point represents the mean \pm S.D. of 3 samples. $* p<0.05$, significant difference from sennoside A (Dunnett's test). 
Table 1. Within-Day and Day-to-Day Relative Standard Deviations for Sennoside A in Standard Solution II and Daiokanzoto with Fecal Suspension

\begin{tabular}{lccc}
\hline \hline & \multirow{2}{A}{$\begin{array}{c}\text { Amount } \\
(\mathrm{mm})\end{array}$} & Within-day $^{a)}$ & Day-to-day $\left.^{b}\right)$ \\
\cline { 3 - 4 } & & 0.25 & 0.71 \\
& 0.206 & 1.04 & 1.31 \\
\hline Standard solution II & 0.203 & & \\
Daiokanzoto & & & \\
\hline
\end{tabular}

a) $n=15, b) n=5$.

activity of sennoside A metabolism was significantly accelerated by increasing the amounts of liquiritin and liquiritin apioside (Figs. 3C, D). These are flavonoid glycosides abundantly contained in glycyrrhiza. These results indicated that glycyrrhiza significantly enhanced the transformation of sennoside A. Liquiritin and liquiritin apioside contributed as active substances in glycyrrhiza, which promoted the metabolism of sennoside A.

Kampo medicines are orally administered so that the glycosides contained in them may be metabolized by the intestinal bacteria before absorption from the gastrointestinal tract. DKT composed of rhubarb and glycyrrhiza is one of the most frequently prescribed Kampo medicines and is used for the treatment of constipation. The purgative effect of DKT is due to rhubarb. Sennoside A, the main purgative constituent of rhubarb, is a prodrug that is transformed into an active form, rheinanthrone, by intestinal bacteria. In the present study, we investigated the finding that sennoside A was significantly metabolized in the presence of glycyrrhiza. Furthermore, the metabolic activity of sennoside A was significantly accelerated when liquiritin or liquiritin apioside coexisted with sennoside $\mathrm{A}$ in a dose-dependent manner.

In summary, the HPLC method described in this report is sufficiently reliable and simple to be used for the determination of sennoside $\mathrm{A}$ in an incubation mixture of DKT with mouse feces. We demonstrate that liquiritin and liquiritin apioside significantly enhance the potent transformation of sennoside A using this method. The results of this study sug- gest that the influence of these constituents on the fate of rheinanthrone transformed from sennoside A improve the purgative activity of rhubarb. Therefore, we consider that the significance of the combination of rhubarb and glycyrrhiza has been proved in the purgative action of DKT.

Acknowledgements The authors are grateful to Ms. Kiyoka Kashihara, Mr. Takuya Sakamoto, Mr. Shota Nakagawa, Ms. Yuka Mitsuki, Ms. Aya Yabuuchi, Ms. Ayaka Yamane and Mr. Kazuya Yoshifuji for their technical assistance.

\section{References}

1) Kobashi K., Akao T., Bioscience Microflora, 16, 1-7 (1997).

2) Kim D.-H., Jung E.-A., Sohng I.-S., Han J.-A., Kim T. H., Han M. J., Arch. Pharm. Res., 21, 17-23 (1998).

3) Kim D.-H., Nat. Prod. Sci., 8, 35-43 (2002).

4) "Ippanyo Kampo Syoho No Tebiki," ed. by Supervision by the Pharmaceutical and Supply Bureau of Health and Welfare Ministry of Japan, Yakugyo Jihosya, Tokyo, 1976.

5) Miyawaki Y., Chin M., Yagi T., Yamauchi K., Kuwano S., J. Med. Pharm. Soc., Wakan-Yaku, 10, 97-103 (1993).

6) Yagi T., Yamauchi K., J. Trad. Med, 18, 191-196 (2001).

7) Sasaki K., Yamauchi K., Kuwano S., Planta Med., 37, 370-378 (1979).

8) Kobashi K., Nishimura T., Kusaka M., Hattori M., Namba T., Planta Med., 40, 225-236 (1980).

9) Lemi J., Lemmens L., Pharmacology, 20, 50—57 (1980).

10) Kobayashi T., Kohata M., Takemura N., Okamura N., J. Trad. Med., 26, 80-85 (2009).

11) Oshima Y., Takahashi K., Hiraga Y., Shibata S., Shoyakugaku Zasshi, 37, 217-222 (1983).

12) Oshima T., Hirayama F., Masuda M., Maruta T., Sagara K., Mizutani T., He L.-Y., Tong Y.-Y., Chen Y.-H., Itokawa H., J. Chromatogr. A, 585, 255-258 (1991).

13) Koyama J., Morita I., Fujiyoshi H., Kobayashi N., Chem. Pharm. Bull., 53, 573-575 (2005).

14) Komatsu K., Nagayama Y., Tanaka K., Ling Y., Basnet P., Meselhy M. R., Chem. Pharm. Bull., 54, 941-947 (2006).

15) Okamura N., Maki T., Miyauchi H., Shimoe M., Yokono S., Yoshitomi H., Yagi A., Biol. Pharm. Bull., 24, 1161-1164 (2001).

16) Okamura N., Miyauchi H., Choshi T., Ishizu T., Yagi A., Biol. Pharm. Bull., 26, 658-661 (2003).

17) Tokuyama K., Umeno M., Okamura N., J. Trad. Med., 22, 252-256 (2005).

18) Okamura N., Abo N., Aono M., Yagi A., Nat. Med., 56, 1-6 (2002). 